\title{
SMOOTH VALUES OF THE ITERATES OF THE EULER'S PHI-FUNCTION
}

\author{
Youness LAMZOURI
}

\begin{abstract}
Let $\phi(n)$ be the Euler-phi function, define $\phi_{0}(n)=n$ and $\phi_{k+1}(n)=\phi\left(\phi_{k}(n)\right)$ for all $k \geq 0$. We will determine an asymptotic formula for the set of integers $n$ less than $x$ for which $\phi_{k}(n)$ is $y$-smooth, conditionally on a weak form of the Elliott-Halberstam conjecture.
\end{abstract}

\section{INTRODUCTION}

Integers without large prime factors, usually called smooth numbers, play a central role in several topics of number theory. From multiplicative questions to analytic methods, they have various and wide applications, and understanding their behavior will have important consequences for number theoretic algorithms, which are an important tool in cryptography.

Let $\phi(n)$ be the Euler-phi function, define $\phi_{0}(n)=n$ and $\phi_{k+1}(n)=\phi\left(\phi_{k}(n)\right)$ for all $k \geq 0$. There are several interesting results on the behavior of the functions $\phi_{k}$ (Erdös, Granville, Pomerance and Spiro [5]). It is known that the understanding of the multiplicative structure of the phi-function and its iterates is in some sense equivalent to studying the behavior of the integers of the form $p-1$ where $p$ is prime. It is also believed that the distribution of the prime factors of such an integer behaves like that of a random integer, in the following sense: Define

$$
\Psi(x, y)=|\{n \leq x: p \mid n \Longrightarrow p \leq y\}| \quad \text { and } \quad \pi(x, y)=|\{p \leq x: q \mid p-1 \Longrightarrow q \leq y\}| .
$$

Conjecture 1. Fix $U \geq 1$. If $x^{1 / U} \leq y \leq x$ then

$$
\frac{\pi(x, y)}{\pi(x)} \sim \frac{\Psi(x, y)}{x} \quad \text { as } \quad x \rightarrow \infty .
$$

Assuming this conjecture one can deduce the behavior of the function $\pi(x, y)$ from the known asymptotic formula

$$
\Psi(x, y) \sim x \rho(u) \text { as } x \rightarrow \infty \text { with } x=y^{u}
$$

AMS subject classification: 11N37, 11B37, 34K05, 45J05. 
where $\rho(u)$ is the Dickman function, defined as the unique continuous solution of the differential-difference equation $u \rho^{\prime}(u)=-\rho(u-1)$ for $u \geq 1$, satisfying the initial condition $\rho(u)=1$ for $0 \leq u \leq 1$.

Now let $P$ be a set of prime numbers and define

$$
\Psi(x, P)=|\{n \leq x: p \mid n \Longrightarrow p \in P\}| \quad \text { and } \quad \pi(x, P)=|\{p \leq x: q \mid p-1 \Longrightarrow q \in P\}| \text {. }
$$

One might guess

$$
\frac{\pi(x, P)}{\pi(x)} \sim \frac{\Psi(x, P)}{x} \quad \text { as } \quad x \rightarrow \infty,
$$

under certain conditions on the set $P$.

Granville [7] has an unpublished argument that Conjecture 1 holds for $u=\log (x) / \log (y)$ bounded, assuming the Elliott-Halberstam conjecture (E-H) which states that:

$$
\sum_{q \leq x^{1-\epsilon}} \max _{y \leq x} \max _{(a, q)=1}\left|\pi(y ; q, a)-\frac{\pi(y)}{\phi(q)}\right| \ll_{\epsilon, A} \frac{x}{\log (x)^{A}} .
$$

A weak version of this conjecture is the following:

Conjecture 2. Fix $\epsilon>0$. Then

$$
\sum_{d \leq x^{1-\epsilon}}\left|\pi(x ; d, 1)-\frac{\pi(x)}{\phi(d)}\right|=o(\pi(x)) \quad \text { as } \quad x \rightarrow \infty .
$$

We will prove a version of (1) assuming this Conjecture; specifically we show the following:

Theorem 1. Assume Conjecture 2. If $P$ is a set of primes less than $x$ for which

$$
\sum_{\substack{p \notin P \\ p \leq x}} \frac{1}{p} \ll 1 \quad \text { then } \quad \frac{\pi(x, P)}{\pi(x)} \sim \prod_{p \notin P}\left(1-\frac{1}{(p-1)^{2}}\right) \frac{\Psi(x, P)}{x} \quad \text { as } x \rightarrow \infty .
$$

Note that there is an extra factor in Theorem 1 compared with (1). To see why we should expect this, let $q$ be some prime; then the probability that a random integer $n$ is divisible by $q$ is $1 / q$. Now the probability that a random integer of the form $p-1$ (where $p$ prime) is divisible by $q$ is $1 /(q-1)$ (since $p$ is excluded from the class $0 \bmod q$ ). The differences between the two probabilities are negligible as $q$ increases, however this is not true for small primes $q$, and thus we need a correction factor (it can be removed in some special cases, see Lemma 2.1).

Define

$$
\Phi_{k}(x, y)=\left|\left\{n \leq x: p \mid \phi_{k}(n) \Longrightarrow p \leq y\right\}\right| .
$$

Using Theorem 1 we get an asymptotic of this function conditionally on conjecture 2 . 
Theorem 2. Assume Conjecture 2. Fix $U>1$. If $y=x^{1 / u}$ where $1 \leq u \leq U$, then

$$
\Phi_{k}(x, y) \sim x \sigma_{k}(u) \quad \text { as } \quad x \rightarrow \infty
$$

where $\sigma_{k}(u)=1$ for $u \leq 1$, and $u \sigma_{k+1}(u)=\int_{0}^{u} \sigma_{k+1}(u-t) \sigma_{k}(t) d t$ for $u \geq 1$, with $\sigma_{0}(u)=$ $\rho(u)=((e+o(1)) / u \log (u))^{u}$. Moreover, for all $k \geq 1$

$$
\sigma_{k}(u)=\left(\frac{1+o(1)}{\log _{k}(u) \log _{k+1}(u)}\right)^{u} \text { and } \log _{k}(u)=\log (\log (\log (\ldots \log (u) \ldots))) \quad k \text { times. }
$$

The first step in the proof uses simple combinatorics to approximate the functions $\Phi_{k}(x, y)$ by $\Psi\left(x, P_{k}\right)$, where $P_{k}$ are the sets of primes defined iteratively by $P_{k+1}=\{p \leq$ $\left.x: q \mid p-1 \Longrightarrow q \in P_{k}\right\}$, with $P_{0}=\{p \leq y\}$.

\section{Proposition 1.}

$$
\Phi_{k}(x, y)=\Psi\left(x, P_{k}\right)+O\left(\frac{x(\log x)^{2 k}}{y}\right)
$$

From the fact that $\left|P_{k}\right|=\pi\left(x, P_{k-1}\right)$, the next step in proving our Theorem 2 is to establish a relation between $|P|$ and $\Psi(x, P)$ for any given set of primes $P$. This was done by Granville and Soundararajan [8] while studying mean values of multiplicative functions. They proved the following proposition:

Proposition 2 (Proposition 1 of [8]). Let $f$ be a multiplicative function with $|f(n)| \leq 1$ for all $n$, and $f(n)=1$ for $n \leq y$. Let $\theta(x)=\sum_{p \leq x} \log (p)$ and define

$$
\chi(u):=\frac{1}{\theta\left(y^{u}\right)} \sum_{p \leq y^{u}} f(p) \log (p) .
$$

Then $\chi(t)$ is a measurable function with $\chi(t)=1$ for all $t \leq 1$. Let $\sigma$ be the corresponding unique solution to the equation:

$$
u \sigma(u)=\int_{0}^{u} \sigma(u-t) \chi(t) d t \quad \text { for } \quad u>1
$$

subject to the initial condition $\sigma(u)=1$ for $0 \leq u \leq 1$. Then

$$
\frac{1}{y^{u}} \sum_{n \leq y^{u}} f(n)=\sigma(u)+O\left(\frac{u}{\log (y)}\right) .
$$

From this result and by partial summation we can deduce 
Corollary 1. Fix $U>1$. Let $P$ be a set of primes less than $x$ such that $P_{0} \subseteq P$, and $f$ be a completely multiplicative function such that $f(p)=1$ if $p \in P$ and 0 otherwise (so that $f(n)=1$ for all $n \leq y)$. For $1 \leq u \leq U$, define

$$
\chi(u):=\frac{1}{\pi\left(y^{u}\right)} \sum_{\substack{p \in P \\ p \leq y^{u}}} 1,
$$

then

$$
\Psi\left(y^{u}, P\right)=\sum_{n \leq x} f(n) \sim y^{u} \sigma(u)
$$

where $\sigma$ is the corresponding solution to (2).

It remains to study (2), a delay integral equation, and to try to estimate the solution $\sigma$ where $\chi$ is a certain measurable function. In several interesting cases $\chi(u)$ decays like $(\{1+o(1)\} / h(u))^{u}$ where $h$ is positive and non-decreasing. We prove the following:

Theorem 3. Let $\chi$ be a real measurable function for which $\chi(t)=1$ for $0 \leq t \leq 1$, and $0 \leq \chi(t) \leq 1$ for $t>1$. Moreover suppose that

i) $\int_{T}^{\infty} \chi(t) d t=0$ for some constant $T$. We define $T=\min \left\{t: \int_{T}^{\infty} \chi(t) d t=0\right\}$ to avoid redundancy, and suppose that $T>1$.

or ii) $\chi(t)=(\{1+o(1)\} / h(t))^{t}$ where $h(t)$ is non-decreasing and $h(t) \rightarrow \infty$ as $t \rightarrow \infty$.

Let $\sigma$ be the corresponding solution to (2). Then

$$
\sigma(u)=\exp \left((-\xi(u)+o(1)) u+\int_{1}^{\infty} \frac{\chi(v) e^{\xi(u) v}}{v} d v\right)
$$

where $\xi(u)$ is the unique solution to $u=\int_{1}^{\infty} \chi(v) e^{\xi(u) v} d v$.

Moreover we can get explicit asymptotic in a number of interesting cases, we prove

Proposition 3. Let $\chi$ be a real measurable function for which $\chi(t)=1$ for $0 \leq t \leq 1$, and $0 \leq \chi(t) \leq 1$ for $t>1$. Suppose that $\int_{T}^{\infty} \chi(t) d t=0$ for some constant $T$. We define $T=\min \left\{t: \int_{T}^{\infty} \chi(t) d t=0\right\}$ to avoid redundancy, and suppose that $T>1$. Then $\xi(u)=\frac{\log (u)}{T}(1+o(1))$, and

$$
\sigma(u)=\exp \left(-\frac{u \log (u)}{T}(1+o(1))\right)
$$

Proposition 4. Let $\chi$ be a real measurable function for which $\chi(t)=1$ for $0 \leq t \leq 1$, and $0 \leq \chi(t) \leq 1$ for $t>1$; and suppose that $\chi(u)=(\{1+o(1)\} / h(u))^{u}$ where $h$ satisfies the following conditions:

i) $h$ is positive and non-decreasing with $h(u) \rightarrow \infty$ as $u \rightarrow \infty$. 
ii) $h$ is continuously differentiable and $u h^{\prime}(u) / h(u) \rightarrow n$ as $u \rightarrow \infty$ for some $0 \leq n<\infty$.

We distinguish two cases: a) $0<n<\infty$ and b) $n=0$. Then

$$
\sigma(u)=\left(\frac{1+o(1)}{h(\zeta \log (u))}\right)^{u}
$$

where $\zeta=e / n$ in case a) and $\zeta=1$ in case $b)$.

The distinction between cases a) and b) in Proposition 4 justifies the appearance of the constant $e$ only in the asymptotic of $\sigma_{o}$ in Theorem 2 .

\section{Acknowledgments.}

I sincerely thank my advisor, Professor Andrew Granville, for all his advices and encouragements. I would also thank the referee for his rigorous comments and remarks.

\section{Proof of Theorem 2}

Lemma 2.1. Assume Conjecture 2. Fix $U \geq 1$. Suppose that $P$ is a set of primes less than $x$ for which $\{p \leq y\} \subseteq P$, where $y=x^{1 / u}$ and $1 \leq u \leq U$. Then

$$
\frac{\pi(x, P)}{\pi(x)} \sim \frac{\Psi(x, P)}{x} \text { as } \quad x \rightarrow \infty .
$$

Proof. We have that

$$
\sum_{\substack{p \notin P \\ p \leq x}} \frac{1}{p} \leq \sum_{y<p \leq x} \frac{1}{p} \ll \log \left(\frac{\log (x)}{\log (y)}\right)=\log (u) \ll 1
$$

and, since $1-t \geq e^{-2 t}$ for $0 \leq t \leq 1 / 2$, then

$$
1 \geq \prod_{p \notin P}\left(1-\frac{1}{(p-1)^{2}}\right) \geq \prod_{p>y}\left(1-\frac{1}{(p-1)^{2}}\right) \geq \exp \left(-2 \sum_{p>y} \frac{1}{(p-1)^{2}}\right)=1+o(1) .
$$

The result follows by Theorem 1 .

Proof of Theorem 2. First note that the sets $P_{k}$ for $k \geq 0$ satisfy the conditions of Lemma 2.1. Now $\Psi\left(x, P_{0}\right)=\Psi(x, y) \sim \rho(u) x$ as $x \rightarrow \infty$. We use induction on $k$ : suppose that $\Psi\left(x, P_{k}\right) \sim \sigma_{k}(u) x$ as $x \rightarrow \infty$ for some smooth function $\sigma_{k}(u)$; then by Lemma 2.1

$$
\frac{\left|P_{k+1}\right|}{\pi(x)}=\frac{\pi\left(x, P_{k}\right)}{\pi(x)} \sim \frac{\Psi\left(x, P_{k}\right)}{x} \sim \sigma_{k}(u) \text { as } x \rightarrow \infty .
$$

Now by corollary 1 we have

$$
\Psi\left(x, P_{k+1}\right) \sim \sigma_{k+1}(u) x \text { as } x \rightarrow \infty,
$$

where $\sigma_{k+1}(u)$ is the corresponding solution to $(2)$ with $\chi(u)=\sigma_{k}(u)$. Noting that $\sigma_{0}(u)=$ $\rho(u)=((e+o(1)) / u \log (u))^{u}$ and using proposition 4 we deduce that

$$
\sigma_{k}(u)=\left(\frac{1+o(1)}{\log _{k}(u) \log _{k+1}(u)}\right)^{u}
$$

by induction. Thus, using proposition 1 , the Theorem follows. 


\section{Proof of Theorem 1}

Lemma 3.1. If $P$ is a set of primes $\leq x$, then

$$
\sum_{\substack{p \notin P \\ p \leq x}} \frac{1}{p} \ll 1 \quad \Longleftrightarrow \quad \prod_{p \in P}\left(1-\frac{1}{p}\right) \asymp \frac{1}{\log (x)} .
$$

Proof. The result follows since

$$
\prod_{p \in P}\left(1-\frac{1}{p}\right)=\prod_{\substack{p \notin P \\ p \leq x}}\left(1-\frac{1}{p}\right)^{-1} \prod_{p \leq x}\left(1-\frac{1}{p}\right) \asymp \exp \left(O\left(\sum_{\substack{p \notin P \\ p \leq x}} \frac{1}{p}\right)\right) \frac{1}{\log (x)}
$$

by Mertens theorem.

Lemma 3.2. Let $m$, $d$ be positive integers such that $d \mid m$, then we have

$$
\sum_{\substack{r \leq x \\ d|r| m}} \frac{\mu(r)}{r}=\mu(d) \sum_{\substack{n \geq 1 \\ d|n \\ p| n}} \frac{1}{n} \sum_{\substack{r \leq x / n \\ r \mid m}} \frac{\mu(r)}{r} .
$$

Proof. The result is trivial if $\mu(d)=0$ or $d=1$. We fix $m$ and do a double induction on $d \geq 1$ and $x \geq 1$. Now

$$
\begin{aligned}
S_{d}(x) & :=\sum_{\substack{r \leq x \\
d|r| m}} \frac{\mu(r)}{r}=\sum_{\substack{n \leq x / d \\
n \mid \frac{m}{d}}} \frac{\mu(d n)}{d n}=\frac{\mu(d)}{d} \sum_{\substack{n \leq x / d \\
n \mid m \\
(n, d)=1}} \frac{\mu(n)}{n}=\frac{\mu(d)}{d} \sum_{\substack{n \leq x / d \\
n \mid m}} \frac{\mu(n)}{n} \sum_{\substack{a|n \\
a| d}} \mu(a) \\
& =\frac{\mu(d)}{d} \sum_{a \mid d} \mu(a) \sum_{\substack{n \leq x / d \\
a|n| m}} \frac{\mu(n)}{n}=\frac{\mu(d)}{d} \sum_{a \mid d} \mu(a) S_{a}(x / d) .
\end{aligned}
$$

Now each $a \leq d$ and $x / d<x$ so, by induction

$$
\begin{aligned}
& S_{d}(x)=\frac{\mu(d)}{d} \sum_{a \mid d} \mu(a)^{2} \sum_{\substack{n \geq 1 \\
a|n \\
p| n \Longrightarrow p \mid a}} \frac{1}{n} \sum_{\substack{r \leq x / n d \\
r \mid m}} \frac{\mu(r)}{r} \\
& =\frac{\mu(d)}{d} \sum_{\substack{n \geq 1 \\
p|n \Longrightarrow p| d}} \frac{1}{n} \sum_{\substack{r \leq x / n d \\
r \mid m}} \frac{\mu(r)}{r} \sum_{\substack{a|d \\
a| n \\
a \mid n}}^{\Longrightarrow p \mid a}
\end{aligned}
$$


Now if we write $n=p_{1}^{b_{1}} p_{2}^{b_{2}} \ldots . p_{k}^{b_{k}}$ with each $b_{j} \geq 1$, then $p|n \Longrightarrow p| d$ implies that $p_{1} p_{2} \ldots p_{k} \mid d$. Moreover if $a$ satisfies $a|d, a| n, p|n \Longrightarrow p| a$, and $a$ is a squarefree, then $a$ must be $p_{1} p_{2} \ldots p_{k}$; which implies

$$
\sum_{\substack{a|d \\ a| n \\ p|n \stackrel{n}{\Longrightarrow} p| a}} \mu(a)^{2}=1 .
$$

Then, writing $l=n d$, we have $S_{d}(x)=\mu(d) \sum_{\substack{l \geq 1 \\ p|l| l \\ p|l| l}} \frac{1}{l} \sum_{\substack{r \leq x / l \\ r \mid m}} \frac{\mu(r)}{r}$, as desired.

Lemma 3.3. For any positive integer $k$ we have

$$
\sum_{\substack{n \geq 1 \\ k \backslash n \\ p \mid n}} \frac{\log (n)}{n}=\frac{1}{\phi(k)}\left(\sum_{p \mid k} \frac{\log (p)}{p-1}+\log (k)\right) \asymp \frac{\log (k)}{\phi(k)} .
$$

Proof. Writing $n=k d$ we have

$$
\sum_{\substack{n \geq 1 \\ k \backslash n \\ k \mid n}} \frac{\log (n)}{n}=\sum_{\substack{d \geq 1 \\ p|d| k}} \frac{\log (d)+\log (k)}{d k}=\frac{1}{k} \sum_{\substack{d \geq 1 \\ p|d \stackrel{\text {. }}{\Longrightarrow} p| k}} \frac{\log (d)}{d}+\frac{\log (k)}{\phi(k)} .
$$

Now if $p_{1}, p_{2}, \ldots, p_{n}$ are the prime factors of $k$ then

$$
\begin{aligned}
& \sum_{\substack{d \geq 1 \\
p|d \stackrel{\text { a }}{\Longrightarrow} p| k}} \frac{\log (d)}{d}=\sum_{\substack{a_{i} \geq 0 \\
1 \leq i \leq n}} \frac{a_{1} \log \left(p_{1}\right)+a_{2} \log \left(p_{2}\right)+\ldots+a_{n} \log \left(p_{n}\right)}{p_{1}^{a_{1}} p_{2}^{a_{2}} \ldots p_{n}^{a_{n}}} \\
= & \sum_{i=1}^{n}\left(\sum_{a_{i} \geq 0} \frac{a_{i} \log \left(p_{i}\right)}{p_{i}^{a_{i}}}\right)\left(\prod_{\substack{1 \leq j \leq n \\
j \neq i}}\left(\sum_{a_{j} \geq 0} \frac{1}{p_{j}^{a_{j}}}\right)\right)=\sum_{i=1}^{n} \frac{\log \left(p_{i}\right)}{p_{i}\left(1-\frac{1}{p_{i}}\right)^{2}} \prod_{\substack{1 \leq j \leq n \\
j \neq i}}\left(1-\frac{1}{p_{j}}\right)^{-1} \\
= & \frac{k}{\phi(k)} \sum_{p \mid k} \frac{\log (p)}{p-1}, \text { which gives the result. }
\end{aligned}
$$

We state a classical result of Sieve theory which is used throughout the proof: 
Lemma 3.4 (Brun's Sieve). Let $A$ be a set of positive integers contained in $[1, N]$. Suppose that for each prime $p \leq N, A$ is excluded from $\omega(p)$ residue classes mod $p$, where $\omega$ is a multiplicative function and $\omega(p) \ll 1$. Then

$$
|A| \ll N \prod_{p \leq N}\left(1-\frac{\omega(p)}{p}\right) .
$$

Proof of Theorem 1. Let $\epsilon>0, P^{*}=\{p \leq x\} \backslash P$, and $m=\prod_{p \in P^{*}} p$. Then we have

$$
\begin{aligned}
\pi(x, P) & =\sum_{\substack{p \leq x \\
q \mid p-1 \stackrel{\Longrightarrow}{\Longrightarrow} q \in P}} 1=\sum_{\substack{p \leq x \\
(p-1, m)=1}} 1=\sum_{p \leq x} \sum_{d \mid(m, p-1)} \mu(d)=\sum_{d \mid m} \mu(d) \sum_{\substack{p \leq x \\
d \mid p-1}} 1 \\
& =\sum_{d \mid m} \mu(d) \pi(x ; d, 1) .
\end{aligned}
$$

Now by a similar argument we have

$$
\Psi(x, P)=\sum_{\substack{d \leq x \\ d \mid m}} \mu(d)\left[\frac{x}{d}\right] .
$$

By (3) and assuming Conjecture 2 we have

$$
\pi(x, P)=\left(\sum_{\substack{d \leq x^{1-\epsilon} \\ d \mid m}} \frac{\mu(d)}{\phi(d)}\right) \pi(x)+O\left(\sum_{\substack{x^{1-\epsilon}<d \leq x \\ d \mid m}} \pi(x ; d, 1)\right)+o(\pi(x)) .
$$

From (4), lemmas 3.1 and 3.4 we deduce

$$
\left|\Psi(x, P)-x \sum_{\substack{d \leq x \\ d \mid m}} \frac{\mu(d)}{d}\right| \leq \sum_{\substack{d \leq x \\ d \mid m}} 1 \leq \sum_{\substack{d \leq x \\ p \mid d \stackrel{\text { ․․ }}{\Longrightarrow} p \notin P}} 1 \ll x \prod_{p \in P}\left(1-\frac{1}{p}\right) \ll \frac{x}{\log (x)} .
$$

Also by lemmas 3.1 and 3.4 we have

$$
\begin{aligned}
\sum_{\substack{x^{1-\epsilon}<d \leq x \\
d \mid m}} \frac{1}{d} \leq \sum_{\substack{x^{1-\epsilon}<d \leq x \\
p \mid d \Longrightarrow p \in P^{*}}} \frac{1}{d}=\int_{x^{1-\epsilon}}^{x} \frac{d \Psi\left(t, P^{*}\right)}{t} \leq \frac{\Psi\left(x, P^{*}\right)}{x}+\int_{x^{1-\epsilon}}^{x} \frac{\Psi\left(t, P^{*}\right)}{t^{2}} d t \\
\ll \prod_{p \in P}\left(1-\frac{1}{p}\right)\left(1+\int_{x^{1-\epsilon}}^{x} \frac{d t}{t}\right) \ll \prod_{p \in P}\left(1-\frac{1}{p}\right) \epsilon \log (x) \ll \epsilon .
\end{aligned}
$$


Then from (5), (6) and (7) we deduce

$$
\begin{aligned}
& \left|\frac{\pi(x, P)}{\pi(x)}-\prod_{p \notin P}\left(1-\frac{1}{(p-1)^{2}}\right) \frac{\Psi(x, P)}{x}\right| \\
& \leq\left|\sum_{\substack{d \leq x^{1-\epsilon} \\
d \mid m}} \frac{\mu(d)}{\phi(d)}-\sum_{\substack{d \leq x^{1-\epsilon} \\
d \mid m}} \frac{\mu(d)}{d} \prod_{p \notin P}\left(1-\frac{1}{(p-1)^{2}}\right)\right| \\
& +o(1)+O(\epsilon)+O\left(\sum_{\substack{x^{1-\epsilon}<d \leq x \\
d \mid m}} \frac{\pi(x ; d, 1)}{\pi(x)}\right) .
\end{aligned}
$$

Now by lemmas $3.1,3.4$, and the fact that $\sum_{r \leq x} \frac{1}{\phi(r)} \ll \log (x)$ we get

$$
\begin{aligned}
& \sum_{\substack{x^{1-\epsilon}<d \leq x \\
d \mid m}} \pi(x ; d, 1)=\sum_{\substack { r \leq x^{\epsilon} \\
\begin{subarray}{c}{1-\epsilon \\
p \mid d \leq d \leq x / r \\
d r+1 p r i m e{ r \leq x ^ { \epsilon } \\
\begin{subarray} { c } { 1 - \epsilon \\
p | d \leq d \leq x / r \\
d r + 1 p r i m e } }\end{subarray}} \sum_{\substack{r \leq x^{\epsilon} \\
\text { prime }}} 1 \ll \sum_{p \in P} \frac{x}{r}\left(1-\frac{1}{p}\right) \prod_{\substack{p \leq x \\
p \nmid r}}\left(1-\frac{1}{p}\right) \\
& \ll \frac{x}{\log (x)^{2}} \sum_{r \leq x^{\epsilon}} \frac{1}{\phi(r)} \ll \epsilon \frac{x}{\log (x)} .
\end{aligned}
$$

And from lemma 3.2 we have

$$
\begin{aligned}
& \sum_{\substack{d \leq x^{1-\epsilon} \\
d \mid m}} \frac{\mu(d)}{\phi(d)}=\sum_{\substack{d \leq x^{1-\epsilon} \\
d \mid m}} \frac{\mu(d)}{d} \sum_{k \mid d} \frac{\mu(k)^{2}}{\phi(k)}
\end{aligned}
$$

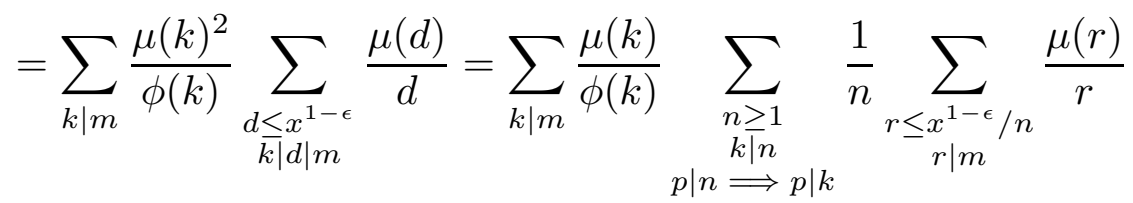

$$
\begin{aligned}
& =\sum_{k \mid m} \frac{\mu(k)}{\phi(k)} \sum_{\substack{n \geq 1 \\
k|n \\
p| n \Longrightarrow p \mid k}} \frac{1}{n} \sum_{\substack{r \leq x^{1-\epsilon} \\
r \mid m}} \frac{\mu(r)}{r}-\sum_{k \mid m} \frac{\mu(k)}{\phi(k)} \sum_{\substack{n \geq 1 \\
k|n \\
p| n \stackrel{n}{\Longrightarrow} \frac{1}{n}}} \sum_{\substack{x^{1-\epsilon} / n<r \leq x^{1-\epsilon} \\
r \mid m}} \frac{\mu(r)}{r} .
\end{aligned}
$$

The first term in the RHS of (10) is equal to:

$$
\sum_{\substack{r \leq x^{1-\epsilon} \\ r \mid m}} \frac{\mu(r)}{r} \sum_{k \mid m} \frac{\mu(k)}{k \phi(k)} \prod_{p \mid k}\left(1-\frac{1}{p}\right)^{-1}=\sum_{\substack{r \leq x^{1-\epsilon} \\ r \mid m}} \frac{\mu(r)}{r} \prod_{p \mid m}\left(1-\frac{1}{(p-1)^{2}}\right)
$$


By integration by parts and using lemma 3.4 we have

$$
\begin{aligned}
\sum_{\substack{x^{1-\epsilon} / n<r \leq x^{1-\epsilon} \\
r \mid m}} \frac{\mu(r)}{r} \mid & \leq \sum_{\substack{x^{1-\epsilon} / n<r \leq x^{1-\epsilon} \\
r \mid m}} \frac{1}{r} \leq \int_{x^{1-\epsilon} / n}^{x^{1-\epsilon}} \frac{d \Psi\left(t, P^{*}\right)}{t} \\
& \ll \prod_{p \in P}\left(1-\frac{1}{p}\right)\left(1+\int_{x^{1-\epsilon} / n}^{x^{1-\epsilon}} \frac{d t}{t}\right) \ll \frac{\log (n)}{\log (x)} .
\end{aligned}
$$

Then, by lemma 3.3

$$
\begin{aligned}
& \sum_{k \mid m} \frac{\mu(k)}{\phi(k)} \sum_{\substack{n \geq 1 \\
k|n \\
p| n \stackrel{n}{\Longrightarrow} p \mid k}} \frac{1}{n} \sum_{\substack{x^{1-\epsilon} / n<r \leq x^{1-\epsilon} \\
r \mid m}} \frac{\mu(r)}{r} \ll \sum_{k \mid m} \frac{\mu(k)}{\phi(k)} \sum_{\substack{n \geq 1 \\
k|n \\
p| n}} \frac{\log (n)}{n \log (x)} \\
& \ll \frac{1}{\log (x)} \sum_{k \mid m} \frac{\mu(k) \log (k)}{\phi(k)^{2}} \ll \frac{1}{\log (x)} .
\end{aligned}
$$

Thus combining (8), (9), (10), (11) and (12) gives the result, letting $\epsilon \rightarrow 0$.

\section{Proof of Proposition 1}

Lemma 4.1. $P_{0}=\{p \leq y\} \subseteq P_{1} \subseteq P_{2} \subseteq \ldots \subseteq P_{k} \subseteq$.. where $P_{k+1}=\{$ primes $q \leq x$ : $\left.p \mid q-1 \Longrightarrow p \in P_{k}\right\}$.

Proof. If $p \in P_{0}$ then $p \leq y$ and so $p-1 \leq y$, which implies $q \mid p-1 \Longrightarrow q \leq y$. This means that $p \in P_{1}$. Now using a simple induction argument: if $p \in P_{k}$ then $q \mid p-1 \Longrightarrow$ $q \in P_{k-1} \subseteq P_{k}$, and so $p \in P_{k+1}$.

Lemma 4.2. Let $r$ be a positive integer. Then

$$
R(r, k, x):=\sum_{\substack{y<r<q_{1}<\ldots<q_{k} \leq x \\ r\left|q_{1}-1, q_{1}\right| q_{2}-1, \ldots, q_{k-1} \mid q_{k}-1}} \frac{1}{q_{k}} \leq \frac{(\log x+1)^{k}}{r} .
$$

We deduce that

$$
S(r, k, x):=\sum_{\substack{y<r<q_{1}<\ldots<q_{k} \leq n \leq x \\ r\left|q_{1}-1, q_{1}\right| q_{2}-1, \ldots, q_{k-1}\left|q_{k}-1, q_{k}\right| n}} 1 \leq \frac{x(\log x+1)^{k}}{r} .
$$

Proof. Writing $q_{k}-1=m q_{k-1}$ we have

$$
R(r, k, x) \leq \sum_{m \leq \frac{x}{r}} \frac{1}{m} R(r, k-1, x) \leq R(r, k-1, x)(\log x+1),
$$


and

$$
R(r, 1, x) \leq \sum_{m \leq \frac{x}{r}} \frac{1}{m r} \leq \frac{\log x+1}{r}
$$

then by induction

$$
R(r, k, x) \leq \frac{(\log x+1)^{k}}{r}
$$

The second inequality follows since

$$
S(r, k, x) \leq \sum_{\substack{y<r<q_{1}<\ldots<q_{k} \leq x \\ r\left|q_{1}-1, q_{1}\right| q_{2}-1, \ldots, q_{k-1} \mid q_{k}-1}} \frac{x}{q_{k}}=x R(r, k, x) .
$$

\section{Lemma 4.3. Define}

$$
S_{k}(x, y)=\left\{n \leq x \text { : there is a prime } p>y \text { such that } p^{2} \mid \phi_{k}(n)\right\}
$$

Then

$$
\left|\Psi\left(x, P_{k}\right)-\Phi_{k}(x, y)\right| \leq \sum_{i=0}^{k-1}\left|S_{i}(x, y)\right|
$$

Proof. Let $A_{k}(x)=\left\{n \leq x: p \mid n \Longrightarrow p \in P_{k}\right\}$. If $n \in A_{k+1}(x)$ and $\phi(n) \notin A_{k}(x)$, then there is a prime $p$ which divides $\phi(n)$ and $p \notin P_{k}$. Now $n \in A_{k+1}(x)$ so every prime factor of $q-1$, where $q \mid n$, is in $P_{k}$, which implies that $p^{2} \mid n$. This gives

$A_{k+1}(x) \backslash\left\{n \leq x: \phi(n) \in A_{k}(x)\right\}=\left\{n \leq x: n \in A_{k+1}(x), \exists\right.$ a prime $\left.p \in P_{k+1} \backslash P_{k}, p^{2} \mid n\right\}$.

Then by lemma 4.1

$$
\begin{aligned}
0 & \leq \Psi\left(x, P_{k}\right)-\Phi_{k}(x, y)=\left|A_{k}(x)\right|-\left|\left\{n \leq x: \phi_{k}(n) \in A_{0}(x)\right\}\right| \\
& =\sum_{i=0}^{k-1}\left|\left\{n \leq x: \phi_{i}(n) \in A_{k-i}(x)\right\}\right|-\left|\left\{n \leq x: \phi_{i+1}(n) \in A_{k-i-1}(x)\right\}\right| \\
& =\sum_{i=0}^{k-1} \mid\left\{n \leq x: \phi_{i}(n) \in A_{k-i}(x), \text { there is a prime } p \in P_{k-i} \backslash P_{k-i-1}, p^{2} \mid \phi_{i}(n)\right\} \mid \\
& \leq \sum_{i=0}^{k-1}\left|S_{i}(x, y)\right| .
\end{aligned}
$$

Proof of Proposition 1. Note that if $q \mid(\phi(n), n)$ for some prime $q$, then $q^{2} \mid n$. Define

$$
S_{k}^{*}(x, y)=S_{k}(x, y) \backslash \bigcup_{i=0}^{k-1} S_{i}(x, y)
$$


If $n \in S_{k}^{*}(x, y)$ and $q^{2} \mid \phi_{j}(n)$ for some $0 \leq j \leq k-1$, then $q \leq y$ (by definition); also there exists some prime $p$ satisfying $p^{2} \mid \phi_{k}(n)$ with $p>y$, which implies $p^{2} \nmid \phi_{k-1}(n)$. Thus we have two cases :

(i) There exists a prime $q_{1} \mid \phi_{k-1}(n)$ such that $p^{2} \mid q_{1}-1$.

(ii) There are two primes $q_{1} \mid \phi_{k-1}(n)$ and $Q_{1} \mid \phi_{k-1}(n)$ such that $p \mid q_{1}-1$ and $p \mid Q_{1}-1$.

In the first case $q_{1}\left|\phi_{k-1}(n)=\phi\left(\phi_{k-2}(n)\right), p\right| q_{1}-1$, so that $q_{1}>y$, which implies that $q_{1}^{2} \nmid \phi_{k-2}(n)$, so that there exists a prime $q_{2} \mid \phi_{k-2}(n)$ such that $q_{1} \mid q_{2}-1$ and $q_{2}>q_{1}>$ $p>y$. By a simple induction, there exist primes $y<p<q_{1}<q_{2}<\ldots<q_{k}$ for which $p^{2}\left|q_{1}-1, q_{1}\right| q_{2}-1, \ldots, q_{k-1}\left|q_{k}-1, q_{k}\right| n$.

We deduce that the total number of possibilities for this case is:

$$
S_{1}=\sum_{\substack{y<p<q_{1}<\ldots<q_{k} \leq n \leq x \\ p^{2}\left|q_{1}-1, q_{1}\right| q_{2}-1, \ldots, q_{k-1}\left|q_{k}-1, q_{k}\right| n}} 1=\sum_{y<p<\sqrt{x}} S\left(p^{2}, k, x\right) \leq x(\log x+1)^{k} \sum_{p>y} \frac{1}{p^{2}} \ll \frac{x(\log x)^{k}}{y}
$$

by lemma 4.2 .

Now following an analogous argument we find (for the second case) that there exist primes $p, q_{1}, q_{2}, \ldots, q_{k}, Q_{1}, Q_{2}, \ldots, Q_{k}$ such that $p\left|q_{1}-1, q_{1}\right| q_{2}-1, \ldots, q_{k-1}\left|q_{k}-1, q_{k}\right| n$ and $p\left|Q_{1}-1, Q_{1}\right| Q_{2}-1, \ldots, Q_{k-1}\left|Q_{k}-1, Q_{k}\right| n$; we'll have two cases again:

a) $q_{i} \neq Q_{i}$ for all $1 \leq i \leq k$.

b) There exists $i$ such that $q_{i}=Q_{i}$; so let $j=\min \left\{1 \leq i \leq k: q_{i}=Q_{i}\right\}$.

For case a) the total number of possibilities is:

$$
\begin{aligned}
& S_{2}=\sum_{\substack{y<p<q_{1}<\ldots<q_{k} \leq n \leq x \\
p<Q_{1}<\ldots<Q_{k} \leq n \leq x \\
p\left|q_{1}-1, q_{1}\right| q_{2}-1, \ldots, q_{k-1}\left|q_{k}-1, q_{k}\right| n \\
p\left|Q_{1}-1, Q_{1}\right| Q_{2}-1, \ldots, Q_{k-1}\left|Q_{k}-1, Q_{k}\right| n}} 1 \leq \sum_{\begin{array}{c}
y<p<q_{1}<\ldots<q_{k} \leq x \\
p<Q_{1}<\ldots<Q_{k} \leq x \\
p\left|q_{1}-1, q_{1}\right| q_{2}-1, \ldots, q_{k-1} \mid q_{k}-1 \\
p\left|Q_{1}-1, Q_{1}\right| Q_{2}-1, \ldots, Q_{k-1} \mid Q_{k}-1
\end{array}} \frac{x}{q_{k} Q_{k}} \\
& \leq x \sum_{p>y} R(p, k, x)^{2}=O\left(\frac{x(\log x)^{2 k}}{y}\right)
\end{aligned}
$$

by lemma 4.2 .

Now for case b) $p\left|q_{1}-1, q_{1}\right| q_{2}-1, \ldots, q_{j-1}\left|q_{j}-1, p\right| Q_{1}-1, Q_{1}\left|Q_{2}-1, \ldots, Q_{j-1}\right| Q_{j}-1$ and $Q_{j}=q_{j} \mid \phi_{k-j}(n)$, then following the same logic there exist primes $q_{j+1}, q_{j+2}, \ldots, q_{k}$ such that $q_{j}\left|q_{j+1}-1, \ldots, q_{k-1}\right| q_{k}-1, q_{k} \mid n$. 
We deduce that the total number of possibilities is:

$$
\begin{aligned}
& S_{3}=\sum_{\substack{y<p<q_{1}<\ldots<q_{j} \leq x \\
p\left|q_{1}-1, q_{1}\right| q_{2}-1, \ldots, q_{j-1} \mid q_{j}-1}}\left(\sum_{\substack{p<Q_{1}<\ldots<Q_{j}=q_{j} \\
p\left|Q_{1}-1, Q_{1}\right| Q_{2}-1, \ldots, Q_{j-1} \mid Q_{j}-1}}\left(\sum_{\substack{q j<q_{j+1}<\ldots<q_{k} \leq n \leq x \\
q_{j}\left|q_{j+1}-1, \ldots q_{k}-1\right| q_{k}-1, q_{k} \mid n}} 1\right)\right) \\
& =\sum_{\substack{y<p<q_{1}<\ldots<q_{j} \leq x \\
p\left|q_{1}-1, q_{1}\right| q_{2}-1, \ldots, q_{j-1} \mid q_{j}-1}}\left(\sum_{\substack{p<Q_{1}<\ldots<Q_{j}=q_{j} \\
p\left|Q_{1}-1, Q_{1}\right| Q_{2}-1, \ldots, Q_{j-1} \mid Q_{j}-1}} S\left(q_{j}, k-j, x\right)\right) \\
& \leq \sum_{\substack{y<p<q_{1}<\ldots<q_{j} \leq x \\
p\left|q_{1}-1, q_{1}\right| q_{2}-1, \ldots, q_{j-1} \mid q_{j}-1}}\left(\sum_{\substack{p<Q_{1}<\ldots<Q_{j}=q_{j} \\
p\left|Q_{1}-1, Q_{1}\right| Q_{2}-1, \ldots, Q_{j-1} \mid Q_{j}-1}} \frac{x(\log x+1)^{k-j}}{q_{j}}\right) .
\end{aligned}
$$

Now writing $Q_{j}-1=q_{j}-1=m Q_{j-1} q_{j-1}$ we have:

$$
\sum_{\substack{q_{j} \leq x \\ q_{j-1}, Q_{j-1} \mid q_{j}-1}} \frac{1}{q_{j}}<\sum_{m \leq x} \frac{1}{m Q_{j-1} q_{j-1}} \leq \frac{\log x+1}{Q_{j-1} q_{j-1}} .
$$

Thus by lemma 4.2

$$
S_{3} \ll x(\log x)^{k-j+1} \sum_{p>y} R(p, j-1, x)^{2} \ll \frac{x(\log x)^{k+j-1}}{y} .
$$

We deduce from cases (i), (ii) a) and b) that

$$
\left|S_{k}^{*}(x, y)\right|=S_{1}+S_{2}+S_{3}=O\left(\frac{x(\log x)^{2 k}}{y}\right) .
$$

Now

$$
\begin{aligned}
\left|S_{1}(x, y)\right|= & \left|S_{0}(x, y)\right|+\left|S_{1}^{*}(x, y)\right|=\mid\left\{n \leq x: \exists \text { a prime } p>y, p^{2} \mid n\right\} \mid+O\left(\frac{x(\log x)^{2}}{y}\right) \\
\leq & \sum_{\substack{n \leq x \\
p>y \\
p^{2} \mid n}} 1+O\left(\frac{x(\log x)^{2}}{y}\right) \leq \sum_{p>y} \frac{x}{p^{2}}+O\left(\frac{x(\log x)^{2}}{y}\right) \\
= & O\left(\frac{x}{y}\right)+O\left(\frac{x(\log x)^{2}}{y}\right)=O\left(\frac{x(\log x)^{2}}{y}\right),
\end{aligned}
$$

and by simple induction we obtain:

$$
\left|S_{k}(x, y)\right|=\left|S_{k}^{*}(x, y)\right|+\sum_{i=0}^{k-1}\left|S_{i}(x, y)\right|=O\left(\frac{x(\log x)^{2 k}}{y}\right) .
$$

Thus by (14) and lemma 4.3 the result follows. 


\section{Proof of Theorem 3}

Lemma 5.1. Let $\chi$ be a real measurable function for which $\int_{1}^{\infty} \chi(t) e^{\xi t} d t$ converges for all $\xi$ and such that $C:=\int_{1}^{\infty} \chi(v) d v>0$. Then for $u \geq C^{2}$ and for any $\epsilon>0$, we have

$$
\int_{1}^{\infty} \chi(v) e^{(\xi(u)+\epsilon) v} d v \geq u^{1+\frac{\epsilon}{2 \xi(u)}}
$$

Proof. Let $\epsilon^{\prime}>0$ and $s>0$. Using Hölder inequality we get

$$
\left(\int_{1}^{\infty} \chi(v) d v\right)^{\epsilon^{\prime}}\left(\int_{1}^{\infty} \chi(v) e^{s v} d v\right)^{1-\epsilon^{\prime}} \geq \int_{1}^{\infty} \chi(v) e^{s\left(1-\epsilon^{\prime}\right) v} d v
$$

Now putting $s=\xi(u) /\left(1-\epsilon^{\prime}\right)=\xi(u)\left(1+\epsilon^{\prime \prime}\right)$ and since $u \geq C^{2}$ we deduce that

$$
\int_{1}^{\infty} \chi(v) e^{\xi(u)\left(1+\epsilon^{\prime \prime}\right) v} d v \geq \frac{1}{C^{\epsilon^{\prime}}}\left(\int_{1}^{\infty} \chi(v) e^{\xi(u) v} d v\right)^{1+\epsilon^{\prime \prime}} \geq u^{1+\epsilon^{\prime \prime} / 2}
$$

The lemma follows taking $\epsilon=\xi(u) \epsilon^{\prime \prime}$.

Proof of Theorem 3.

The Upper bound.

From Lemma 3.4 of Granville-Soundararajan [8] we note that

$$
\sigma(u)=\rho(u)+\sum_{j=1}^{\infty} \frac{1}{j !} \int_{t_{1}+\cdots+t_{j} \leq u} \frac{\chi\left(t_{1}\right)}{t_{1}, \ldots, t_{j} \geq} \ldots \frac{\chi\left(t_{j}\right)}{t_{j}} \rho\left(u-t_{1}-\ldots-t_{j}\right) d t_{1} \ldots d t_{j}
$$

Therefore for any $\xi \in \mathbb{R}$

$$
\begin{aligned}
\sigma(u) e^{\xi u} & =\rho(u) e^{\xi u}+\sum_{j=1}^{\infty} \frac{1}{j !} \\
& \int_{\substack{t_{1}, \ldots, t_{j} \geq 1 \\
t_{1}+\cdots+t_{j} \leq u}} \frac{\chi\left(t_{1}\right) e^{\xi t_{1}}}{t_{1}} \ldots \frac{\chi\left(t_{j}\right) e^{\xi t_{j}}}{t_{j}} \rho\left(u-t_{1}-\ldots-t_{j}\right) e^{\xi\left(u-t_{1}-\ldots-t_{j}\right)} d t_{1} \ldots d t_{j} .
\end{aligned}
$$

Setting $F(\xi)=\max _{t \geq 0} \rho(t) e^{\xi t}$ we deduce that (by forgetting the condition $t_{1}+\ldots+t_{j} \leq u$ )

$$
\sigma(u) \leq F(\xi) e^{-\xi u} \sum_{j=0}^{\infty} \frac{1}{j !}\left(\int_{1}^{\infty} \frac{\chi(t) e^{\xi t}}{t} d t\right)^{j}=F(\xi) e^{-\xi u} \exp \left(\int_{1}^{\infty} \frac{\chi(t) e^{\xi t}}{t} d t\right)
$$

Choose $\xi$ such that $u=\int_{1}^{\infty} \chi(t) e^{\xi t} d t$, that is $\xi=\xi(u)$. 
Now putting $C:=\int_{1}^{\infty} \chi(v) d v$ we have $u=\int_{1}^{\infty} \chi(v) e^{\xi(u) v} d v \geq C e^{\xi(u)}$, which implies that

$$
F(\xi(u)) \leq \max _{t \geq 0}\left(\frac{(e+o(1)) u}{t \log (t) C}\right)^{t}=e^{O(u / \log (u))},
$$

and the upper bound follows.

The Lower bound. Fix $\epsilon>0$.

We will show that there exists a constant $C_{\epsilon}$ such that

$$
\sigma(u)>C_{\epsilon} \exp \left((-\xi(u)-\epsilon) u+\int_{1}^{\infty} \frac{\chi(v) e^{\xi(u) v}}{v} d v\right) \text { for all } u \geq 0 .
$$

Let $u_{0}$ be a suitably large number, and define

$$
C_{\epsilon}=C_{\epsilon, u_{0}}=\inf _{u \leq u_{0}} \sigma(u) \exp \left((\xi(u)+\epsilon) u-\int_{1}^{\infty} \frac{\chi(v) e^{\xi(u) v}}{v} d v\right) .
$$

Evidently (15) holds for all $u \leq u_{0}$.

We use an induction argument. Let $n \in \mathbb{N}$ such that $n>u_{0}$ and suppose that (15) is verified for all $t \leq n$, then we will show that (15) holds for all $t \in[n, n+1]$.

Define $f(\xi)=\int_{1}^{\infty} \frac{\chi(v) e^{\xi v}}{v} d v$, and let $u \in[n, n+1]$. Then using our hypothesis we have

$$
\frac{\sigma(u) e^{((\xi(u)+\epsilon) u)}}{C_{\epsilon} \exp (f(\xi(u)))}=\frac{1}{C_{\epsilon} u} \int_{0}^{u} \chi(t) e^{(\xi(u)+\epsilon) t} \sigma(u-t) e^{((\xi(u)+\epsilon)(u-t)-f(\xi(u))} d t
$$

$$
\geq \frac{1}{u} \int_{1}^{u} \chi(t) \exp ((\xi(u)+\epsilon) t+(\xi(u)-\xi(u-t))(u-t)+f(\xi(u-t))-f(\xi(u))) d t .
$$

Since $f^{\prime}(\xi)=\int_{1}^{\infty} \chi(v) e^{\xi v} d v$ and using the mean value theorem we deduce that

$$
u-t \leq \frac{f(\xi(u))-f(\xi(u-t))}{\xi(u)-\xi(u-t)} \leq u .
$$

Now differentiating $u=\int_{1}^{\infty} \chi(v) e^{\xi(u) v} d v$ with respect to $u$ we get that

$$
\xi^{\prime}(u)=\left(\int_{1}^{\infty} v \chi(v) e^{\xi(u) v} d v\right)^{-1} \leq \frac{1}{u} .
$$

By (18) and using the mean value theorem again we have

$$
\xi(u)-\xi(u-t) \leq \frac{t}{u-t} .
$$


Therefore by (17), then (19) we deduce that

$$
\begin{aligned}
\frac{1}{u} \int_{1}^{u} \chi(t) & \exp ((\xi(u)+\epsilon) t+(\xi(u)-\xi(u-t))(u-t)+f(\xi(u-t))-f(\xi(u))) d t \\
& \geq \frac{1}{u} \int_{1}^{u} \chi(t) \exp ((\xi(u)+\epsilon) t-t(\xi(u)-\xi(u-t))) d t \\
& \geq \frac{1}{u} \int_{1}^{u} \chi(t) \exp \left((\xi(u)+\epsilon) t-\frac{t^{2}}{(u-t)}\right) d t \\
& \geq \frac{1}{u} \int_{1}^{\sqrt{u}} \chi(t) e^{(\xi(u)+\epsilon / 2) t} d t, \text { for } u \geq u_{0} .
\end{aligned}
$$

For case i), Since $\int_{T}^{\infty} \chi(t) d t=0$ and $\chi(t) \geq 0$ for all $t$, then meas $\{t \geq T: \chi(t) \neq 0\}=0$ which implies that meas $\left\{t \geq T: \chi(t) e^{\xi(u) t} \neq 0\right\}=0$, and so $\int_{T}^{\infty} \chi(t) e^{\xi(u) t} d t=0$. Then taking $u_{0}>T^{2}$ we have

$$
\int_{1}^{\sqrt{u}} \chi(t) e^{(\xi(u)+\epsilon / 2) t} d t=\int_{1}^{T} \chi(t) e^{(\xi(u)+\epsilon / 2) t} d t>\int_{1}^{T} \chi(t) e^{\xi(u) t} d t=u .
$$

Now for case ii) since $\chi(t)=(\{1+o(1)\} / h(t))^{t}$, there exist two constants $A_{\epsilon}$ and $B_{\epsilon}$ for which

$$
A_{\epsilon}\left(\frac{\exp (-\epsilon / 16)}{h(t)}\right)^{t}<\chi(t)<B_{\epsilon}\left(\frac{\exp (\epsilon / 16)}{h(t)}\right)^{t} \text { for every } t \geq 0 .
$$

we consider two cases

1). $\frac{e^{\xi(u)}}{h(\sqrt{u})} \geq \exp \left(-\frac{\epsilon}{4}\right)$. Since $h$ is non-decreasing we have by $(22)$

$$
\begin{aligned}
\int_{1}^{\sqrt{u}} \chi(t) e^{(\xi(u)+\epsilon / 2) t} d t & \geq A_{\epsilon} \int_{1}^{\sqrt{u}}\left(\frac{e^{(\xi(u)+7 \epsilon / 16)}}{h(t)}\right)^{t} d t \geq A_{\epsilon} \int_{1}^{\sqrt{u}}\left(\frac{e^{\xi(u)}}{h(\sqrt{u})}\right)^{t} e^{(7 \epsilon / 16) t} d t \\
& \geq A_{\epsilon} \int_{1}^{\sqrt{u}} e^{(3 \epsilon / 16) t} d t=\frac{16 A_{\epsilon}}{3 \epsilon}\left(e^{(3 \epsilon / 16) \sqrt{u}}-e^{3 \epsilon / 16}\right)>u,
\end{aligned}
$$

for $u>u_{0}$.

2). $\frac{e^{\xi(u)}}{h(\sqrt{u})} \leq \exp \left(-\frac{\epsilon}{4}\right)$. Using lemma 5.1 and $(22)$, then the fact that $h$ is nondecreasing and $u \geq C e^{\xi(u)}$ we conclude that

$$
\begin{aligned}
\int_{1}^{\sqrt{u}} \chi(t) e^{(\xi(u)+\epsilon / 2) t} d t & \geq \int_{1}^{\sqrt{u}} \chi(t) e^{(\xi(u)+\epsilon / 8) t} d t \\
& \geq u^{1+\epsilon /(16 \xi(u))}-B_{\epsilon} \int_{\sqrt{u}}^{\infty}\left(\frac{e^{(\xi(u)+3 \epsilon / 16)}}{h(t)}\right)^{t} d t \\
& \geq u^{1+\epsilon /(16 \xi(u))}-B_{\epsilon} \int_{\sqrt{u}}^{\infty}\left(\frac{e^{\xi(u)}}{h(\sqrt{u})}\right)^{t} e^{(3 \epsilon / 16) t} d t \\
& \geq u^{1+\epsilon /(16 \xi(u))}-B_{\epsilon} \frac{16}{\epsilon} \exp (-\epsilon \sqrt{u} / 16)>u,
\end{aligned}
$$


for $u>u_{0}$. Thus using (16), (20) then (21), (23), and (24) the result follows.

\section{Getting the Asymptotic of $\sigma$ explicithy}

Lemma 6.1. If $\xi(u)=o(\log (u))$ as $u \rightarrow \infty$, then

$$
\int_{1}^{\infty} \frac{\chi(v) e^{\xi(u) v}}{v} d v=o(u) \text { and so } \sigma(u)=\exp ((-\xi(u)+o(1)) u)
$$

Proof. Since $\chi(t) \leq 1$ for every $t \geq 1$ and using our assumption we have

$$
\begin{aligned}
\int_{1}^{\infty} \frac{\chi(v) e^{\xi(u) v}}{v} d v & =\int_{1}^{\frac{\log (u)}{\xi(u)}} \frac{\chi(v) e^{\xi(u) v}}{v} d v+\int_{\frac{\log (u)}{\xi(u)}}^{\infty} \frac{\chi(v) e^{\xi(u) v}}{v} d v \\
& \leq \int_{1}^{\frac{\log (u)}{\xi(u)}} e^{\xi(u) v} d v+\frac{\xi(u)}{\log (u)} \int_{1}^{\infty} \chi(v) e^{\xi(u) v} d v \\
& =\frac{1}{\xi(u)}\left(u-e^{\xi}\right)+\frac{\xi(u) u}{\log (u)}=o(u)
\end{aligned}
$$

Proof of Proposition 3. Let $\zeta(u)$ be the unique continuous solution to the equation $u=$ $e^{\zeta(u) T} / \zeta(u)$. Since $\chi(t) \leq 1$ for all $t$, we have

$$
\frac{e^{\zeta(u) T}}{\zeta(u)}=\int_{1}^{T} \chi(v) e^{\xi(u) v} d v \leq \frac{e^{\xi(u) T}-e^{\xi(u)}}{\xi(u)}<\frac{e^{\xi(u) T}}{\xi(u)},
$$

and since the function $f(\xi)=e^{\xi T} / \xi$ is non-decreasing for $\xi>1$ we deduce that $\zeta(u) \leq \xi(u)$. Now fix $\epsilon>0$ (such that $T(1-\epsilon)>1$ ), and suppose that there is arbitrary large $u$ for which $\xi(u)>\zeta(u)(1+\epsilon)$. Define $s_{\epsilon}=\int_{T(1-\epsilon / 3)}^{T} \chi(t) d t>0$ (by the definition of $T$ ). We deduce under our assumption that

$$
s_{\epsilon} e^{\zeta(u)(1+\epsilon / 3) T} \leq s_{\epsilon} e^{T(1-\epsilon / 3) \xi(u)} \leq \int_{T(1-\epsilon / 3)}^{T} \chi(v) e^{\xi(u) v} d v \leq \frac{e^{\zeta(u) T}}{\zeta(u)}
$$

which is impossible if $u$ is large enough. Thus $\xi(u)=\zeta(u)(1+o(1))$ as $u \rightarrow \infty$. Now we trivially have $1 \ll \zeta(u) \ll \log (u)$, then

$$
\zeta(u)=\frac{\log (u)}{T}+\frac{\log (\zeta(u))}{T}=\frac{\log (u)}{T}(1+o(1)) .
$$

We deduce that $\xi(u)=\frac{\log (u)}{T}(1+o(1))$, and the result follows combining Theorem 3 and the fact that $\int_{1}^{T} \frac{\chi(v) e^{\xi(u) v}}{v} d v=O(u)$.

Now we prove Proposition 4; define $g(u):=h(u) /\left(u h^{\prime}(u)\right)$. 
Lemma 6.2. Let $h(u)$ be a real differentiable function with $u h^{\prime}(u) / h(u)=n+o(1)$, where $n$ is a positive constant. Then for all $k>0$ we have $h(k u)=h(u) k^{n+o(1)}$.

Proof. We have that

$$
\log \left(\frac{h(k u)}{h(u)}\right)=\int_{u}^{k u} \frac{h^{\prime}(t)}{h(t)} d t=\int_{u}^{k u} \frac{(n+o(1))}{t} d t=(n+o(1)) \log k .
$$

Lemma 6.3. Assume the hypothesis of Proposition 4 b).

Then $h(v(u) \log (u))=(1+o(1)) h(\log u)$, where $v(u):=\min \left(\log (u), \min _{\log (u) \leq t \leq \log ^{2}(u)} g(t)\right)$, and $v(u) \rightarrow \infty$ as $u \rightarrow \infty$.

Proof. Since $g(t) \rightarrow \infty$ as $t \rightarrow \infty$ then $v(u) \rightarrow \infty$ as $u \rightarrow \infty$, so if $u$ is large then $v(u) \log (u)>\log u$ so that $h(v(u) \log (u)) \geq h(\log u)$. On the other hand

$$
\begin{aligned}
& \log \left(\frac{h(v(u) \log (u))}{h(\log (u))}\right)=\int_{\log (u)}^{v(u) \log (u)}\left(\frac{h^{\prime}(t)}{h(t)}\right) d t=\int_{\log (u)}^{v(u) \log (u)} \frac{1}{t g(t)} d t \\
& \leq \frac{1}{\min _{\log (u) \leq t \leq v(u) \log (u)} g(t)} \int_{\log (u)}^{v(u) \log (u)} \frac{d t}{t} \leq \frac{1}{v(u)}(1+\log (v(u)))=o(1) .
\end{aligned}
$$

Proof of Proposition 4. Fix $\epsilon>0$ and suppose that there is arbitrary large $u$ for which $\xi(u)>\log (h(\zeta \log (u)))+\epsilon$. Then for such $u$ we have:

In case a) by (22), lemma 6.2 and since $h$ is non-decreasing

$$
\begin{aligned}
u & =\int_{1}^{\infty} \chi(t) e^{\xi(u) t} d t>A_{\epsilon} \int_{1}^{\infty}\left(\frac{e^{\xi(u)-\epsilon / 16}}{h(t)}\right)^{t} d t \geq A_{\epsilon} \int_{\log \log (u)}^{\log (u) / n} e^{(\epsilon / 2) t}\left(\frac{h(e \log (u) / n)}{h(\log (u) / n)}\right)^{t} d t \\
& =A_{\epsilon} \int_{\log \log (u)}^{\log (u) / n} e^{(\epsilon / 2+n+o(1)) t} d t>A_{\epsilon} \int_{\log \log (u)}^{\log (u) / n} e^{(\epsilon / 4+n) t} d t>u,
\end{aligned}
$$

for $u$ large enough, which is a contradiction.

Now in case b), our assumption and lemma 6.3 imply that $\xi(u)>\log (h(v(u) \log (u)))+\epsilon / 2$.

Then by (22) and since $h$ is non-decreasing and $v(u) \rightarrow \infty$ as $u \rightarrow \infty$

$$
\begin{aligned}
u & =\int_{1}^{\infty} \chi(t) e^{\xi(u) t} d t>A_{\epsilon} \int_{1}^{\infty}\left(\frac{e^{\xi(u)-\epsilon / 16}}{h(t)}\right)^{t} d t \geq A_{\epsilon} \int_{1}^{v(u) \log (u)} e^{(\epsilon / 3) t} d t \\
& =A_{\epsilon} \frac{3}{\epsilon}\left(u^{v(u) \epsilon / 3}-e^{\epsilon / 3}\right)>u
\end{aligned}
$$

for $u$ large enough, which is a contradiction. 
Now we suppose that there is arbitrary large $u$ for which $\xi(u)<\log (h(\zeta \log (u)))-\epsilon$. Then for such $u$ let $q(t):=(\xi(u)+\epsilon / 16-\log (h(t))) t$, so that

$$
q^{\prime}(t)=\xi(u)+\frac{\epsilon}{16}-\log (h(t))-\left(\frac{t h^{\prime}(t)}{h(t)}\right) .
$$

Now in case a) $q^{\prime}(t)=\xi(u)+\epsilon / 16-\log (h(t))-n+o(1)$, therefore the maximum of $q(t)$ holds at some point $t_{0}$ for which $q^{\prime}\left(t_{0}\right)=0$ so that, under our assumption

$$
h\left(t_{0}\right)=e^{\xi(u)+\epsilon / 16-n+o(1)}<h(e \log (u) / n) e^{-n-\epsilon / 2} .
$$

Now we must have

$$
t_{0}<\log (u)(1-\epsilon /(4 n)) / n,
$$

otherwise since $h$ is non-decreasing and by lemma 6.2

$\frac{h\left(t_{0}\right)}{h(e \log (u) / n)} \geq \frac{h\left(\frac{\log (u)}{n}\left(1-\frac{\epsilon}{4 n}\right)\right)}{h(e \log (u) / n)}=\left(\left(1-\frac{\epsilon}{4 n}\right) e^{-1}\right)^{n+o(1)}>e^{-n-\epsilon / 2}$ contradicting $(25)$.

By (22), (25) and (26) and since $h$ is non-decreasing we deduce that

$$
\begin{aligned}
u & =\int_{1}^{\infty} \chi(t) e^{\xi(u) t} d t<B_{\epsilon} \int_{1}^{\infty}\left(\frac{e^{\xi(u)+\epsilon / 16}}{h(t)}\right)^{t} d t \\
& =B_{\epsilon} \int_{1}^{e \log (u) / n}\left(\frac{e^{\xi(u)+\epsilon / 16}}{h(t)}\right)^{t} d t+B_{\epsilon} \int_{e \log (u) / n}^{\infty}\left(\frac{e^{\xi(u)+\epsilon / 16}}{h(t)}\right)^{t} d t \\
& \leq B_{\epsilon} \frac{e \log (u)}{n}\left(\frac{e^{\xi(u)+\epsilon / 16}}{h\left(t_{0}\right)}\right)^{t_{0}}+B_{\epsilon} \int_{e \log (u) / n}^{\infty} e^{-\epsilon / 2 t} d t=B_{\epsilon} \frac{e \log (u)}{n}\left(e^{n+o(1)}\right)^{t_{0}}+o(1)<u,
\end{aligned}
$$

for $u$ large enough, which is a contradiction.

For case b) $q^{\prime}(t)=\xi(u)+\epsilon / 16-\log (h(t))-\frac{1}{g(t)}$, and the maximum of $q(t)$ holds at some point $t_{0}$ for which $q^{\prime}\left(t_{0}\right)=0$ (to avoid redundancy we take $t_{0}=\min \left\{t: q^{\prime}(t)=0\right\}$, which is possible by the continuity of $h(t)$ and $g(t))$. Now $t_{0} \rightarrow \infty$ as $u \rightarrow \infty$, otherwise $q^{\prime}\left(t_{0}\right)>0$ for $u$ large enough. Thus

$$
\left(\frac{e^{\xi(u)+\epsilon / 16}}{h\left(t_{0}\right)}\right)^{t_{0}}=\exp \left(\frac{t_{0}}{g\left(t_{0}\right)}\right)=e^{o\left(t_{0}\right)}
$$

Now by $(22)$

$$
\begin{aligned}
u & =\int_{1}^{\infty} \chi(t) e^{\xi(u) t} d t \leq B_{\epsilon} \int_{1}^{\infty}\left(\frac{e^{\xi(u)+\epsilon / 16}}{h(t)}\right)^{t} d t \\
& =B_{\epsilon} \int_{1}^{\log (u)}\left(\frac{e^{\xi(u)+\epsilon / 16}}{h(t)}\right)^{t} d t+B_{\epsilon} \int_{\log (u)}^{\infty}\left(\frac{e^{\xi(u)+\epsilon / 16}}{h(t)}\right)^{t} d t .
\end{aligned}
$$


Considering the cases $t_{0} \leq \log (u)$ and $t_{0}>\log (u)$ (in which case $q(t)$ is increasing on $[1, \log (u)])$, and using $(27)$ and our assumption on $\xi(u)$ we get that

$$
\int_{1}^{\log (u)}\left(\frac{e^{\xi(u)+\epsilon / 16}}{h(t)}\right)^{t} d t \leq \max \left(\log (u) e^{o(\log (u))}, \log (u) \exp (-\epsilon / 2 \log (u))\right)=u^{o(1)}
$$

using this, (28) and the assumption on $\xi(u)$ we deduce that

$$
u \leq B_{\epsilon} u^{o(1)}+B_{\epsilon} \int_{\log (u)}^{\infty} e^{-\epsilon / 2 t} d t=u^{o(1)}+o(1)
$$

which contradicts our hypothesis.

Now in both cases $h^{\prime}(t) / h(t) \leq c / t$ for some positive constant $c$ and for all $t$. Then integrating both sides gives $h(t) \ll t^{c}$, and this with our result implies $\xi(u) \ll \log (\log (u))$. Thus by Lemma 6.1 and Theorem 3 the Proposition follows.

\section{REFERENCES}

[1] R. C. Baker and G. Harman, Shifted primes without large prime factors, Acta Arith. 83, 331-361.

[2] H. Davenport, Multiplicative number theory, 2nd edition, Springer Verlag, New York, 1980.

[3] P. Erdös, Some remarks on the iterates of the $\phi$ and $\sigma$ functions, Colloq.Math. 17, (1967), 195-202.

[4] P. Erdös, On the normal number of prime factors of $p-1$ and some other related problems concerning Euler's $\phi$-function, Quart. J. Math. 6, (Oxford), 205-213.

[5] P. Erdös, A. Granville, C. Pomerance, and C. Spiro, On the normal behavior of the iterates of some arithmetic functions, Analytic Number Theory (Birkhäuser), Boston, (1990), 165-204.

[6] P. Erdös and C. Pomerance, On the normal number of prime factors of $\phi(n)$, Rocky Mountain Math. J. 15, (1985), 343-352.

[7] A. Granville, Smooth numbers: computational number theory and beyond, University of Georgia Mathematics Preprint Series. 13, (2001), 1-52.

[8] A. Granville and K. Soundararajan, The spectrum of multiplicative functions, Annals of Math. 153, (2001), 407-470.

[9] H. Halberstam and H.-E. Richert, Sieve Methods, Academic Press, London, 1974.

[10] A. Hildebrand and G. Tenenbaum, Integers without large prime factors, J. de Théorie des Nombres de Bordeaux 5, 1993.

[11] C. Pomerance, Popular values of Euler's function, Mathematica 27, (1980), 84-89.

[12] G. Tenenbaum, Introduction à la théorie analytique et probabilistique des nombres, Publ. Inst. Elie Cartan 13, 1990.

[13] H. Shapiro, An arithmetic function arising from the $\phi$-function, American Math. Monthly 50, (1943), 18-30.

Départment de Mathématiques et Statistique, Université de Montréal, CP 6128 sucC Centre-Ville, Montréal, QC H3C 3J7, Canada

E-mail address: Lamzouri@dms.umontreal.ca 